\title{
Attribute-specific retroactive inhibition in Peterson and Peterson type short-term memory tasks
}

\author{
RICHARD H. WINNICK \\ University of South Carolina, Columbia, South Carolina 29208
}

\begin{abstract}
Specific interference in a Peterson and Peterson (1959) type short-term memory task with a single attribute of a multidimensional stimulus was demonstrated. This specific interference was created by employing interpolated activity tasks composed of stimuli which were similar to a single attribute of the multidimensional to-be-remembered stimulus. After $8 \mathrm{sec}$ of interpolated activity, memory for the specific dimension which was similar to the interpolated activity task was significantly degraded. The results were taken as supportive evidence for the existence of attribute-specific retroactive inhibition in short-term memory. Implications for the design of interpolated activity tasks and the possibility that separate storage locations exist in short-term memory for the elements of multidimensional stimuli are considered.
\end{abstract}

In a previous study by Winnick and Archer (1974), a color naming task was utilized as the interpolated activity (IA) in a Peterson and Peterson (1959) type short-term memory (STM) procedure. In the study, subjects reported more difficulty in remembering the color attribute of the multidimensional Wisconsin Card Sort Test (WCST) cards than the attributes of the cards' other dimensions. The current study investigates the premise that different types of rest-interval activities may not be interfering equally with memory for all the attributes of the multidimensional to-be-remembered stimulus item. It is hypothesized that IAs which are similar to one dimension of the multidimensional to-be-remembered stimulus will create significantly more interference with the similar dimension than with the other dissimilar dimensions of the stimulus. If this hypothesis is correct, specific interference with a single attribute of a multidimensional to-be-remembered stimulus which will leave the remaining attributes intact should be possible.

\section{METHOD}

\section{Subjects}

The subjects were 54 college student volunteers between the ages of 18 and 25 years. Subjects received no compensation of any type for participation in the study.

\section{Stimuli}

The to-be-remembered stimuli were selected from standard Wisconsin Card Sort Test cards.

Three of the IA tasks were similar to the dimensions of the WCST cards, containing stimuli from the same class, i.e., number, shape, and color. The items were selected to avoid any repetition of the attributes of the dimensions of the WCST cards. The fourth task consisted of dissimilar stimuli of the class "animals." The six stimuli which were repeated twice within the task in a randomly derived order are as follows: number task: 0 , $5,6,7,8,9$; color task: orange, gray, brown, black, pink, tan; shape task: square, rectangle, pentagon, hexagon, octagon, diamond; animal task: dog, bird, cow, frog, turtle, fish.

\section{Apparatus}

The test sequences were projected by a Dunning Anamatic 16-mm strip-film projector onto the $12 \times 12$ in. rear projection screen of a vision tunnel. The subjects' response switches were located at the center front of the vision tunnel, 2 in. apart, and subjects were instructed to use their preferred hand to respond. Stimulus presentation times were controlled by electronic circuitry utilizing Signettics 555 integrated circuitry. All projection, sequencing, and timing apparatus was located in an adjacent room to minimize distractions for the subjects.

\section{Procedure}

Each student was presented with 16 separate test sequences composed of the following events. A single WCST card was projected for $1 \mathrm{sec}$. This duration was empirically determined as allowing the subject time for one overt verbalization of the three attributes of the WCST card, thus maintaining approximately equal rehearsal time for all subjects. The picutre of the WCST card was then followed by $8 \mathrm{sec}$ of interpolated activity. The IA task consisted of 12 successive stimuli presented at the rate of $1 / .66 \mathrm{sec}$. The subject was asked to respond by naming each stimulus object as it appeared. Following the IA, a signal light came on, indicating that three test stimuli representing each of the three attributes of the original stimulus were to be presented separately. Test stimuli used pictorial representations for shape, irregular blobs of color for color, and slash marks $(/ / /)$ to represent numerosity. The subject was required to make a same or different judgment with regard to the test stimulus and the recalled attributes of the original WCST card in that sequence and push the appropriate one of the two response switches as quickly as he could.

\section{Design}

Each subject was presented with 16 sequences of a stimuli in $a$ repeated measures design. The presentation of eight same and eight different discriminations, with each of the four types of IAs appearing twice within both the same and different segments, was controlled by a $16 \times 16$ Latin Square to minimize any possible ordering effects. Within each test sequence, the WCST dimension similar to the IA was tested first, with the remaining two dimensions randomly filling the second and third test positions. For the dissimilar IA task, the WCST dimensions were randomly rotated through the first test position.

\section{RESULTS AND DISCUSSION}

The mean correct responses for dimensions paired with similar vs. dissimilar interpolated activity tasks and for the differing types of interpolated activities are set 
Table 1

Mean Number of Correct Responses as a Function of Type and Class of the Interpolated Activity Task

\begin{tabular}{llc}
\hline $\begin{array}{c}\text { Interpolated } \\
\text { Activity Task }\end{array}$ & $\begin{array}{c}\text { Mean Correct } \\
\text { Responses }\end{array}$ \\
Type & Similar & .86 \\
& Dissimilar & .93 \\
& Color & .89 \\
Class & Shape & .89 \\
& Number & .93 \\
& Animal & .93 \\
\hline
\end{tabular}

out in Table 1. All data were analyzed using a standard repeated measures analysis of variance model as outlined in Edwards (1972).

It can be seen that recall for dimensions paired with similar interpolated activities is significantly degraded from those paired with dissimilar interpolated activities, $\mathrm{F}(2,106)=23.21, \mathrm{p}<.01$.

This finding indicates that it is possible to interfere directly with a single attribute of a multidimensional stimulus. If the multidimensional stimulus "three-red-stars" is followed by an interpolated activity task consisting of color naming, the subject is significantly more likely to remember the dimensions' attributes of "star" and "three" than he is the color dimension's attribute "red." This result suggests the existence of attribute-specific retroactive inhibition in STM.

An implication of the ability to specifically interfere with a single dimension of a multidimensional stimulus is that the attributes are stored independently in STM. It should not have been possible to interfere with a specific portion of stored unit without equally interfering with the entire unit. Whether this separate encoding is simply contiguity based or represents a more elaborate system of reconstruction remains to be investigated.
It can also be seen from Table 1 that different interpolated activities produce differing amounts of interference, even when they are all similar to dimensions of the to-be-remembered materials, $F(3,159)=5.05, p<.01$. It is suggested that this is caused by differences in the difficulty of processing the stimuli of the interpolated activity task, with number and animal being less difficult to process than color and shape.

The results of this study suggest that care should be taken in the design of interpolated activities for use in the Peterson and Peterson (1959) type STM paradigm when multidimensional stimuli are used as to-be-remembered items. If the interpolated activity is similar to a portion of the to-be-remembered material, it may be causing significantly more interference with that portion of the item than with the remaining portions. This effect could cause an increase in the overall forgetting score which would not accurately reflect the subject's ability. It is also likely that this effect causes the subject to guess on the basis of remembered attributes, with the correct/incorrect measure again not accurately reflecting the subject's ability to remember the item. If the interpolated activity task is both similar and difficult to process, the specific interference effect may be compounded. Further research is needed to clarify the effects of interpolated activities in STM paradigms.

\section{REFERENCES}

Edwards, A. L. Experimental design in psychological research. New York: Holt, Rinehart, and Winston, 1972.

Peterson, L. R., \& Peterson, M. J. Short-term retention of individual verbal items. Journal of Experimental Psychology, $1959,58,193-198$

Winnick, R. H., \& Archer, E. J. The retrieval of positive and negative information from short-term memory storage for use in a concept-identification task. Bulletin of the Psychonomic Society, 1974, 3, 309-310.

(Received for publication March 4, 1975.) 